9-1995

\title{
Organization of Sequences of Bifurcations of Periodic Orbits
}

D. A. Sadovskii

J. A. Shaw

John B. Delos

William \& Mary, jbdelos@wm.edu

Follow this and additional works at: https://scholarworks.wm.edu/aspubs

Part of the Physics Commons

\section{Recommended Citation}

Sadovskii, D. A.; Shaw, J. A.; and Delos, John B., Organization of Sequences of Bifurcations of Periodic Orbits (1995). Physical Review Letters, 75(11), 2120-2123.

https://doi.org/10.1103/PhysRevLett.75.2120

This Article is brought to you for free and open access by the Arts and Sciences at W\&M ScholarWorks. It has been accepted for inclusion in Arts \& Sciences Articles by an authorized administrator of W\&M ScholarWorks. For more information, please contact scholarworks@wm.edu. 


\title{
Organization of Sequences of Bifurcations of Periodic Orbits
}

\author{
D. A. Sadovskií, ${ }^{1-3}$ J. A. Shaw, ${ }^{1}$ and J. B. Delos ${ }^{1,2}$ \\ ${ }^{1}$ Department of Physics, College of William \& Mary, Williamsburg, Virginia 23187-8795 \\ ${ }^{2}$ Joint Institute for Laboratory Astrophysics, University of Colorado, Boulder, Colorado 80309-0440 \\ ${ }^{3}$ Université du Littoral, Quai Freycinet 1, B.P. 5526, 59379 Dunkerque Cedex 1, France*
}

(Received 13 April 1995)

\begin{abstract}
Numerical calculations have shown that bifurcations of periodic orbits of Hamiltonian systems often occur in organized groups. Normal-form theory provides an explanation.
\end{abstract}

PACS numbers: 05.45.+b, 03.20.+i, 03.65.Sq, 46.10.+z

The study of periodic orbits (PO's) provides a route to understanding nonintegrable dynamics [1], and periodic orbits have also become central in the study of "quantum chaos" [2]. Classical PO's produce clearly observable effects in atomic spectra: In the case of near-zero-energy states of an atom in magnetic or electric fields, the largescale structure of the absorption spectrum is formed by those short-time orbits of the electron that begin at and return to the nucleus. Many such recurrences have been identified in experimental data [3,4].

If fixed parameters of the system, such as energy or field strength, are made to vary, then the family of periodic orbits changes. For instance, as energy increases new periodic orbits can be created, and as order changes to chaos this results in a proliferation of periodic orbits. In atoms in fields the new orbits are observed as new peaks that emerge in the recurrence spectrum when the scaled energy changes [5]. It follows that the mechanism of individual bifurcations of periodic orbits, and their patterns and sequences (their "organization"), are of fundamental importance to the study of dynamics of both classical and quantum systems.

The standard way to study bifurcations of periodic orbits is to numerically generate trajectories in the neighborhood of a given periodic orbit, and to plot a series of Poincaré surfaces of section (SOS) for different values of the parameter. The SOS method does not, however, address the question of why certain phenomena occur; it gives only "experimental data," i.e., results of purely numerical experiments. Using this method Shaw [6(a)] and Mao and Delos [6(b)] discovered organized sequences of bifurcations associated with the perpendicular orbit of the diamagnetic Kepler problem, discussed below.

In this paper we show how such organized sequences of bifurcations can be understood using "normal-form theory." This theory [1,7-9] is a form of classical perturbation theory that, when combined with the principles of bifurcation theory, gives a qualitative and a quantitative description of motion near a periodic orbit, and of how that motion changes when parameters of the system are made to vary.

We consider the diamagnetic Kepler problem, one electron in highly excited states of energy $E$ in a hydrogen atom in a magnetic field $B$, with Hamiltonian

$$
H=\frac{p_{\rho}^{2}+p_{z}^{2}-\left(\rho^{2}+z^{2}\right)^{-1 / 2}+\rho^{2}}{2}=E B^{-2 / 3}=\varepsilon,
$$

where $\varepsilon$ is the "scaled energy" [6]. This system has an orbit lying in the $z=0$ plane, perpendicular to the applied magnetic field. In rotated semiparabolic coordinates $(\lambda, \sigma)$, such that $(\rho, z)=\left(\lambda^{2}-\sigma^{2}, 2 \lambda \sigma\right)$, this perpendicular orbit lies on the $\lambda$ axis, and the Hamiltonian (1) becomes

$$
\begin{aligned}
H_{\varepsilon}\left(p_{\lambda}, p_{\sigma}, \lambda, \sigma\right)= & \frac{1}{2}\left(p_{\lambda}^{2}+p_{\sigma}^{2}\right)+4\left(\lambda^{2}+\sigma^{2}\right) \\
& \times\left[\frac{1}{2}\left(\lambda^{2}-\sigma^{2}\right)^{2}-\varepsilon\right]=2 .
\end{aligned}
$$

It contains one parameter, the scaled energy $\varepsilon$.

An observation. - Shaw, Mao, and Delos (SMD) [6] were studying the period- 4 bifurcation of the perpendicular orbit, and the role this bifurcation plays in the creation of the new PO that they call "pac-man" [Fig. 1(u)]. A general theorem by Meyer [7] describes generic bifurcations of PO's (Fig. 2). In particular, it asserts that one of the two generic forms of a period- 4 bifurcation has the "islandchain" pattern on the surface of section [Fig. 2(c)]: Four stable PO's ( $O$ points) and four unstable PO's ( $X$ points) move toward the central stable PO, collide with it simultaneously, and disappear, leaving the central PO stable.

SMD saw this phenomenon in their calculations, but they saw that it was part of a more complicated sequence of events (Fig. 1): (a) A stable-unstable pair of period-4 orbits was created nearby in a "saddle-node" bifurcation. (b) A second such pair was created in a similar fashion. (c) The separatrices rearranged into two concentric fourisland chains. (d) The actual period-4 bifurcation of Meyer's theorem occurred: the inner chain shrank and collapsed onto the perpendicular orbit, leaving only the outer chain. All these observations came out of careful examination of many numerical calculations; at the time, SMD could neither anticipate nor explain such sequences of events. They observed, however, that most of the bifurcations of the perpendicular orbit occur through such ordered sequences. We have reasons to believe that these ordered sequences occur in many systems [10]. 

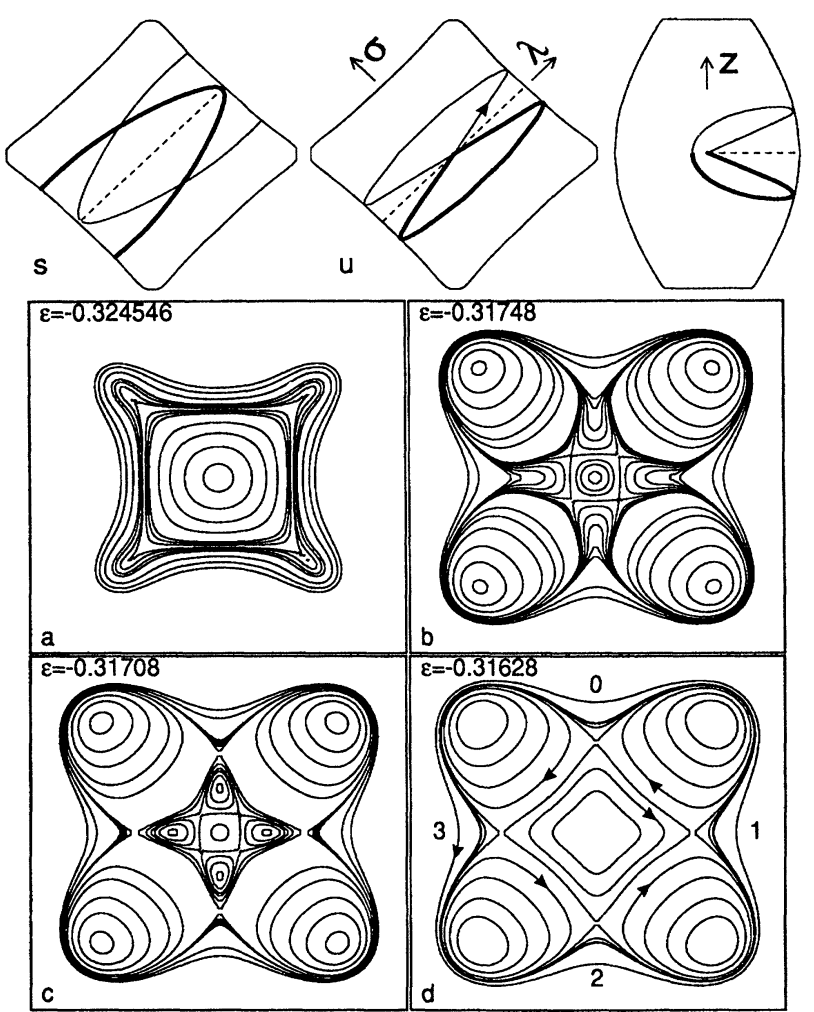

FIG. 1. Top: Periodic trajectories created near the perpendicular orbit after it undergoes a period-4 bifurcation: $(s)$ stable trajectories in $(\lambda, \sigma)$ coordinates; $(u)$ unstable trajectories in $(\lambda, \sigma)$ and in $(\rho, z)$ coordinates for scaled energy $\varepsilon=-0.31$. Bottom: "Organized" sequence of bifurcations near the period- 4 bifurcation of perpendicular orbit.

We use the term organization when we refer to such sequences of bifurcations. It is this kind of phenomenon for which we seek an explanation in normal-form theory.

An analogy. - Consider for a moment a simpler oneparameter Hamiltonian,

$$
\begin{aligned}
\mathcal{H}_{\zeta} & =p_{r}^{2} / 2+V_{\zeta}(r), \\
V_{\zeta}(r) & =\zeta r^{2} / 2-r^{4} / 4+r^{6} / 6 .
\end{aligned}
$$

In this case a bifurcation is any creation or destruction of fixed points of $\mathcal{H}_{\zeta}$, i.e., of points where $p_{r}=\partial V_{\zeta} / \partial r=$ 0 . In Fig. 3 we see that for $\zeta$ large and positive there is a single stable fixed point at $r=0$. As $\zeta$ decreases through $1 / 4$, a new local maximum and minimum of $V_{\zeta}(r)$ are created at a critical point at $r_{0}=1 / \sqrt{2}$. The new unstable and stable fixed points of $\mathcal{H}_{\zeta}$ move apart as $\zeta$ continues to decrease. This is an example of a saddlenode bifurcation [Fig. 2(a)]. Because of the symmetry of (3) about $r=0$ a twin stable-unstable pair appears at the same time at $r_{0}^{\prime}=-1 / \sqrt{2}$. As $\zeta$ decreases through zero, the twin unstable points move to the origin, "collide" with it, and disappear, leaving the origin unstable for negative $\zeta$. This is an example of a "pitchfork" bifurcation [Fig. 2(b)]. (a)
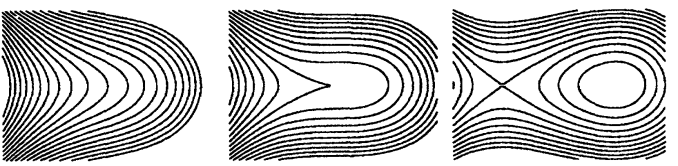

(b)
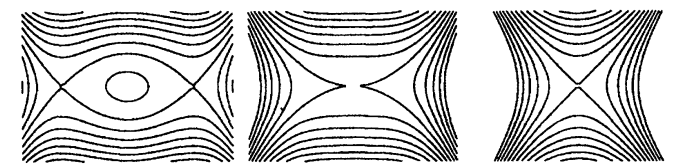

(c)
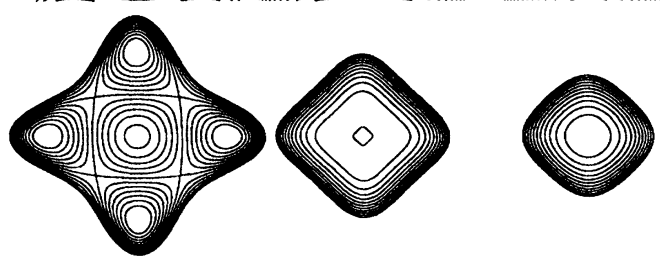

FIG. 2. Generic bifurcations discussed in this Letter: (a) saddle-node (no symmetry), (b) pitchfork (symmetry $C_{2}$ ), and (c) period-4 (symmetry $C_{4}$, case of weak resonance). Curves correspond both to contours of normal forms $\mathcal{H}$ and to interpolated Poincaré surfaces of section.

Both bifurcations of the Hamiltonian (3) follow wellknown patterns. The saddle-node bifurcation (which is generic) has a locally cubic structure $\left(r-r_{0}\right)^{3}-\zeta(r-$ $r_{0}$ ) [Fig. 2(a)], while the pitchfork [generic under the restricting symmetry $V(-r)=V(r)]$ has a locally quartic structure $\zeta r^{2}-r^{4}$ [Fig. 2(b)]. The special point about the Hamiltonian (3) is that it contains these two bifurcations in an organized sequence caused by a monotonic change of single parameter.

Contour plots and the surface of section. - Now consider another Hamiltonian function

$\mathcal{H}_{\varepsilon}(I, \varphi)=\left(\varepsilon-\varepsilon_{4}\right) \alpha I+\left(\beta_{1}+\gamma_{1} \cos 4 \varphi\right) I^{2}+\beta_{2} I^{3}$,

with $\alpha=-1.26, \beta_{1}=-0.11, \gamma_{1}=0.04, \beta_{2}=0.71$, and $\varepsilon_{4} \approx-0.3163$. The important properties of this function are as follows: (i) The coefficient of $I$ passes

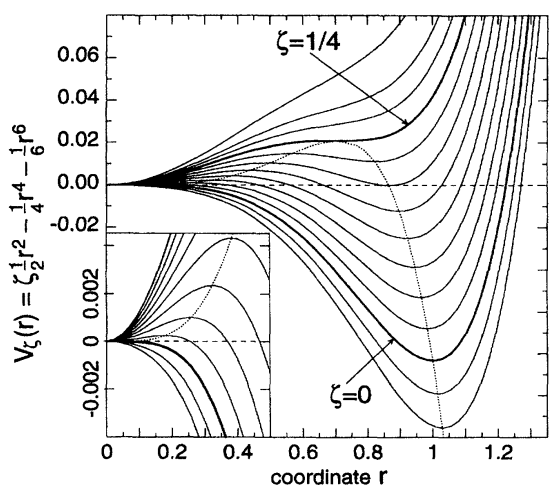

FIG. 3. "Organized" one-parameter bifurcations of stationary points for the function $V_{\zeta}(r)$. Inset: Dotted line gives the position of stationary points. 
through zero at $\varepsilon=\varepsilon_{4}$ while the other coefficients remain nonzero; (ii) $\left|\gamma_{1}\right|<\left|\beta_{1}\right|$, the "case of weak resonance;" and (iii) for any $\varphi$, the coefficients of $I^{2}$ and $I^{3}$ have opposite signs, $\operatorname{sgn}\left(\beta_{1} \pm \gamma_{1}\right)=-\operatorname{sgn}\left(\beta_{2}\right)$.

Hamiltonian (4) has fixed points whenever $\partial H / \partial I=$ $\partial H / \partial \varphi=0$. To find them, note that they must lie on the lines $\varphi=n \pi / 4, n=0, \ldots, 7$. Then note that for any fixed $\varphi$ the substitution $I=r^{2}$ makes the function (4) qualitatively equivalent to $V_{\zeta}(r)$ in Eq. (3b): As $\varepsilon$ increases through $\varepsilon_{4}$, the function $\mathcal{H}_{\varepsilon}(I, \varphi)$ undergoes exactly the same sequence of bifurcations.

Finally, let us define $p_{\sigma}=\sqrt{2 I} \cos \varphi, \sigma=\sqrt{2 I} \sin \varphi$, and let us plot contours of (4) in the $\left(\sigma, p_{\sigma}\right)$ plane. The result is Fig. 1. The contours of this simple function of two variables and one parameter are indistinguishable from the surface-of-section plots obtained laboriously by Shaw, Mao, and Delos [6]. The organized sequence of bifurcations that they found but could not explain is entirely contained in this one function.

Normal-form theory is a systematic procedure for locally converting the original Hamiltonian (2) near a given PO into an effective Hamiltonian like (4). At its lowest level of approximation, retaining only linear and quadratic terms in $I$, it leads to the wonderful theorem of Meyer [7(a)], which asserts that PO's typically bifurcate in just five ways, depending on the period-multiplication factor [11]. At higher levels of approximation (with more terms) this theory leads to effective Hamiltonians that can also describe bifurcations of other related orbits in the greater neighborhood of the original PO, and the sequential organization of these bifurcations.

In this Letter we report the first complete quantitative computer realization of the normal-form expansion about a PO [12]. We have used it to calculate formulas like Eq. (4) and thereby to explain observations of bifurcations near the perpendicular orbit.

Let us see how normal-form theory works and what it contributes by examining two other bifurcations of the perpendicular orbit: a period-3 bifurcation, which occurs when the dynamics is almost regular, and a period-1 bifurcation, which marks the onset of global chaos.

A period-3 bifurcation occurs at a very low value of $\varepsilon\left(\varepsilon_{3} \approx-0.483\right)$. Because of symmetries of the system $[6,11,12]$, this bifurcation produces a six-island chain (Fig. 4). SMD guessed that this chain was the ultimate outcome of a sequence of events similar to the period-4 case in Fig. 1 (outer ring of saddle nodes, inner ring, reconnection, and disappearance of the inner island chain). A numerical search for this sequence of bifurcations was unsuccessful.

Our normal-form calculations [12] showed that the parameter $\beta_{1}$ increases as $\varepsilon$ becomes more negative. Implementing the procedure near the period- 3 bifurcation, we obtained the normal form

$\mathcal{H}_{\varepsilon}(I, \varphi)=\left(\varepsilon-\varepsilon_{3}\right) \alpha I+\beta_{1} I^{2}+\left(\beta_{2}+\gamma_{2} \cos 6 \varphi\right) I^{3}$,

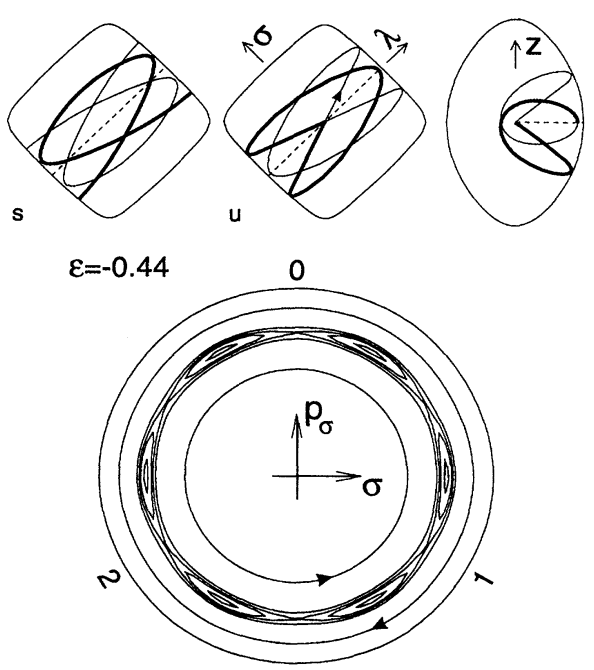

FIG. 4. Top: Periodic trajectories created in the period3 bifurcation: $(u)$ stable trajectories in $(\lambda, \sigma)$ coordinates; $(s)$ unstable trajectories in $(\lambda, \sigma)$ and $(\rho, z)$ coordinates. Bottom: Constant level sections of the $1: 3$ normal form.

with $\alpha=-0.80, \beta_{1}=0.04, \beta_{2}=0.35, \gamma_{2}=0.005$, and $\varepsilon_{3} \approx-0.483$. (The symmetry of the system eliminates the $I^{3 / 2} \cos 3 \varphi$ term, leaving $I^{3} \cos 6 \varphi$ as the main resonance term $[11,12]$.) Here the important point is that the quadratic and cubic terms have the same sign. Therefore the saddle-node bifurcations and the organized sequence are not present. The new island chain is born directly out of the central PO, and steadily moves away as $\varepsilon$ increases.

A period-1 pitchfork bifurcation occurs at $\varepsilon=\varepsilon_{1} \approx$ -0.127 . It is the last bifurcation of the perpendicular orbit: Here this orbit goes unstable, and, as $\varepsilon$ increases further, chaos dominates the phase space. Does this bifurcation happen in the same way as period-4? By close examination of numerical Poincaré surfaces of section, we found a saddle-node bifurcation that created a distant small island of order in the sea of chaos. Our calculations [12] show that the normal form is

$\mathcal{H}_{\varepsilon}\left(p_{\sigma}, \sigma\right)=\frac{1}{2} p_{\sigma}^{2}+\frac{1}{2} k_{1}\left(\varepsilon-\varepsilon_{1}\right) \sigma^{2}+k_{2} \sigma^{4}+k_{3} \sigma^{6}$,

with $k_{1}=-6.84, k_{2}=-0.46$, and $k_{3}=0.40$. Again the important fact is that the $\sigma^{4}$ and $\sigma^{6}$ terms have opposite signs. The consequence is again an organized sequence, this time exactly like that for Hamiltonian (3): Saddlenode bifurcations occur on the $\pm \sigma$ axes; then the newly created unstable points move toward the origin, collide with it, and leave it unstable (Fig. 5).

Comparison with the numerically generated SOS (Fig. 5) displays another important property of the normal-form method: The theory converts the true Hamiltonian, for which the dynamics is almost entirely chaotic, into a different effective Hamiltonian, which has only integrable dynamics. In so doing, it preserves 

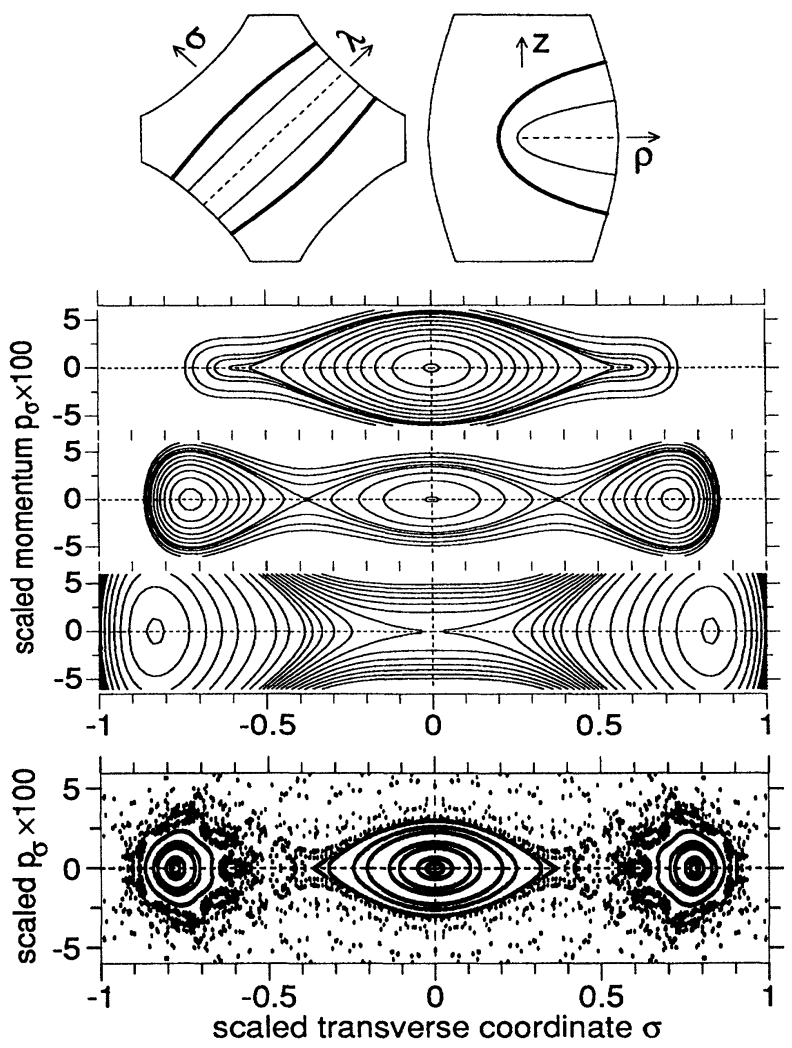

FIG. 5. Top: Periodic trajectories created near the perpendicular orbit before it undergoes its last bifurcation (the period-1 pitchfork) and becomes unstable. Orbits are drawn in $(\lambda, \sigma)$ and $(\rho, z)$ coordinates for scaled energy $\varepsilon=-0.14$. Stable trajectory is shown by a bold line. Center: Contour plot of the normal form $\mathcal{H}_{\varepsilon}\left(\sigma, p_{\sigma}\right)$ near this bifurcation: (descending) $\varepsilon-\varepsilon_{1}=-0.022,-0.015,0.002$. Bottom: Numerical Poincaré surface of section near this bifurcation: $\varepsilon=-0.14$, $\varepsilon-\varepsilon_{1}=-0.01273$.

locally the essential short-time dynamics; i.e., it preserves the structure and stability of nearby short periodic orbits. Normal-form theory replaces the complicated exact dynamics seen in the SOS by contour plots of simple functions, and thereby it provides simple explanations of the organization of sequences of bifurcations in the vicinity of a periodic orbit.

How general is this phenomenon of organization of bifurcations [10]? Let us suppose that the normal form, generally a divergent series, nevertheless provides an adequate representation of the dynamics in the local area of interest around a periodic orbit. The principal order of the phenomenon is $r^{6}\left(I^{3}\right)$, and therefore it will suffice if, for some distance from the central orbit $r_{0}$, terms $r^{8}$ and $r^{10}$ do not result in qualitative changes within $r<r_{0}$. If this holds, the organization we have described occurs whenever terms $r^{4}$ and $r^{6}$ have opposite signs. Therefore these organized sequences of bifurcations must be common.

This work was supported by ONR and NSF through grants to the College of William \& Mary and to JILA. We especially thank JILA for hospitality. We also thank $\mathrm{K}$. Meyer for discussion and inspiration.

*Present address.

[1] H. Poincaré, New Methods of Celestial Mechanics (Dover, New York, 1957), Vol. III; G. D. Birkhoff, Dynamical Systems (American Mathematical Society, New York, 1966).

[2] M.C. Gutzwiller, Chaos in Classical and Quantum Mechanics (Springer, New York, 1991).

[3] M. L. Du and J. B. Delos, Phys. Rev. Lett. 58, 1731 (1987); Phys. Rev. A 38, 1896 (1988); 38, 1913 (1988).

[4] D. Wintgen, Phys. Rev. Lett. 58, 1589 (1987); D. Wintgen and H. Friedrich, Phys. Rev. A 35, 1464 (1987).

[5] J. Main, G. Wiebusch, K. Welge, J. Shaw, and J. B. Delos, Phys. Rev. A 49, 847 (1994).

[6] (a) J. Shaw, Ph.D. thesis, College of William \& Mary, 1993; (b) J.-M. Mao and J. B. Delos, Phys. Rev. A 45, 1746 (1992). The scaling constant used by Mao and Delos differs by $1 / 2:(\hat{\rho}, \hat{z})=(\alpha \rho, \alpha z), \alpha=\frac{1}{2}(B / c)^{2 / 3}$.

[7] (a) K. R. Meyer, Trans. Am. Math. Soc. 149, 95 (1970); 154, 273 (1971); (b) K. R. Meyer and G. R. Hall, Introduction to Hamiltonian Dynamical Systems and the N-Body Problem (Springer, New York, 1992).

[8] A. Deprit, Celest. Mech. 1, 12 (1969).

[9] Vl.I. Arnol'd, Geometrical Methods of the Theory of Ordinary Differential Equations (Springer, New York, 1988); Vl. I. Arnol'd, V. V. Kozlov, and A. I. Neíshtadt, Dynamical Systems III (Springer, New York, 1988).

[10] K. Meyer (private communication) told us about a number of other instances where similar phenomena were observed. He stressed that these phenomena are not generic in the one-parameter theory. See also Table Ic and remarks on pp. 188 and 200 of Ref. [11].

[11] An extension of the generic theory [7(a)] to symmetric systems was given by M. A. M. de Aguiar, C.P. Malta, M. Baranger, and K. T. R. Davies, Ann. Phys. (N.Y.) 180, 167 (1987); M. A. M. de Aguiar and C.P. Malta, Physica (Amsterdam) 30D, 413 (1988).

[12] D. Sadovskií and J.B. Delos (to be published). For quantitative comparisons with exact dynamics, the normal forms need a few more terms; the ones given in this Letter retain only enough terms to give the correct symmetry and qualitative behavior. 